therefore drawn up the accompanying table of the symptoms (1) of acute suppuration in the mastoid bone and (2) of septic thrombos of the lateral sinus. You will, I think, do well to compare the two sets of symptoms carefully, for it is hardly needful to point out that if the sinus is blocked no amount of opening up the mastoid alone will save the patient's life; and, on the other hand, if no blocking of the sinus exists an operation for its exploration is not only unnecessary but may do serious harm.

The table in the main speaks sufficiently for itself. I wish, however, to say a few words concerning two of the symptoms mentioned - viz., the tenderness over the region of the mastoid foramen and the "choked" optic discs. The tenderness over the situation of the mastoid foramen may, as I have already pointed out, be regarded as certain evidence in these cases of blocking of the lateral sinus for the reasons which $I$ have mentioned, excepting in infants and very young children. In such patients it is, of course, impossible to localise the tenderness sufficiently to be of value; moreover, in these very young patients-i.e., before the age of five, or perhaps sixdisease of the masto $d$ is not infrequently associated with subdural abscess, which makes its way to the scalp by coming ont of the sknll through the unossified masto-parietal suture, in which case post-mastoid tenderness occurs independently of blocking of the sinus, which seems less common in ear disease in these very young subjects than in older patients. The value of a knowledge of the importance of this symptom (post-mastoid tenderness) in blocked sinus is well shown by the following case.

On Jan. 23rd, 1893, I was asked to see a girl about fifteen years of age, of whose illness the following history was given. For years she had suffered from time to time from a purulent discharge from the right ear, which had not caused any pain till a week before I saw her, when, after an acute attack of earache, the discharge became more profuse than usual. Two days later, in consequence of the breaking down of a cab, she had to walk about half a mile in the snow at night. On the following day she felt cold and shivered and was almost immediately seized with acute frontal pain and intolerance of light. She was sont to bed and after a severe shivering attack perspired profusely. Some drowsiness followed and continued till I $\$ a w$ her, when her condition was as follows. She was lying in bed in a condition of semi-coma. From this she was easily roused for a short time, during which she spoke cationally and complained of headache, but soon relapsed into the drowsy state. The pulse was very quick and not hard, the temperature was $103^{\circ}$; the face inclined to duskiness. A brightlight in the room caused evident distress. In the right membrana tympani was a large perforation from which a little pus exuded. Hard pressure over the mastoid bone caused the patient to flinch a little, but there was no tenderness at all over the region of the mastoid foramen; the optic discs were normal. Altogether the symptoms simulated in some respects blocking of the lateral sinus as closely as any case of the kind that I had ever seen; but, as you will have noticed, the crucial syraptom-viz., post-mastoid tenderness-was wanting, and to my mind made it perfoctly certain that no surgical interference in the direction of the lateral sinus was indicated. I therefore determined to wait. A day later ulceration on the right side of the fauces and right tonsil developed. The drowsiness and all the other head symptoms gradually passed away spontaneously in the course of the next forty-eight hours, so I left the patient in the hands of the family medical attendant. She subsequently developed pneumonia of a very low type, ber whole illness being dependent probably upon poisoning lsy sewer air, a theory corroborated by the fact that her mother, who had no ear affection or other organic disease, was seized almost directly in the same way and died of pneumonia in seventy-two hours. The case of this patient appears to be an admirable one to illustrate not only the value of a knowledge of the symptom we are now discussing, bnt also the advantage of delay before operating in some of these cases of acute symptoms arising apparently in connexion with ear disease.

With regard to the condition of the optic discs in these cases I have not much to say. "Choked disc" (a term which I prefer to "optic neuritis," as I do not see how the condition can be correctly regarded as inflammatory) is a very uncertain symptom and one concerning which we have much to learn. In thrombus of the lateral sinus it is sometimes absent altogether; in advanced cases it is generally present on both sides, although the sinus is blocked on one side only. It may be unilateral, and when so is occasionally found on the side opposite to that of the affected sinus. The moral of these uncertainties and variations is merely this, that although the presence of choked disc on one or both sides is corroborative of blocking of the lateral sinus its absence in no way negatives the existence of thrombus. Unnecessary delay should not therefore be countenanced in waiting for the manifestation of this symptom before operating, especially as there are, as I have pointed out, other signs by which thrombus in the lateral sinus may be diagnosed with certainty.

\section{DISCOVERY OF THE LIFE HISTORY OF BILHARZIA HAEMATOBIA (COBBOLD).}

BY DR. PROSPERO SONSINO, LECTURER ON PARASITES IN THE UNIVERSITY OF PISA.

HAVING been for a long time desirous of continuing $m y$ studies on the life history of the bilharzia, which were interrupted on my leaving Egypt in 1885, it occurred to me that the south of Tunis would be a suitable place for the purpose. Indeed, the fact that instances of bilharzia disease have recently been discovered in France amongst soldiers who had served in Tunis (Villeneuve's, Brauth's and Calvier's cases) proves that this African parasite is to be found even in the most northern parts of the continent. I arrived in 'Tunis last June, but failed to find examples of bilharzia disease in that city. I was more successful in Gabes, where I found several cases, the material from which enabled me to bring my investigations to a successful conclusion. I cannot say whether bilharzia disease can be acquired in Gabes itself, as the six cases which I saw there were in individuals who had come from the interior of the country. Four of them belonged to one family, the members of which had been living in Gabes for a year, having come from Kbillee (on the eastern side of the Shot of Djereed), and where they were already affected with characteristic bilharzia hæmaturia. Of the other two, one, a T'ripolitan. had lived in many different places in the interior and the other, a boy, came from Gafsa. It is quite possible that at the present time the parasite cannot be acquired at Gabes, in consequence of certain arrangements which have been made by the French authorities in connexion with the water-supply, whereby its pollution is prevented. The six cases, however, which $I$ had at my disposal in Gabes provided me with a supply of ova sufficient for the experimental rearing of embryo bilharzia in association with different kinds of freshwater animals (molluscs and arthropods). After many experiments I succeeded with a small crustacean (amphypoda) and obtained evidence that this same crustacean is an effective intermediary host of bilharzia, and so discovered the secret of the life history of this African parasite. Not being quite satisfied on all points I did not at once publish the discovery, but contented myself, in view of establishing the date of my discovery, with sending a sealed letter on Aug. 3rd to the secretary of the Società Toscana di Scienze Naturali in Pisa. The conclusions therein detailed $I$ have now decided to publish. They were as follows : 1. Bilharzia hæmatobia has a life history differing from the typical one of the digenetic trematodes as represented by fasciola hepatica. 2 Its life history is less complicated than that of the digenetic trematodes; it requires an intermediary host and undergoes a metamorphosis, but there is no alternation of nor asexual generation. 3 . In this way it resembles in its life history the holostomes rather than the distomes. 4. The intermediary host is a small crustacean. 5. The free embryo, which s wims actively about, on encountering the crustacean attacks the latter at a vulnerable point and, by means of the papilla at its head, bores and forces its way into the body of this animal after havirg rid itself of its covering of cilia. Having effected an entrance it proceeds to encyst itself. 6 . The part of the crustacean in which the bilharzia cysts are most frequently located is that corresponding to the first segment and near the eye. 7. The encysted larva, being transferred with the crustacean in drinking water to the human stomach, is then set at liberty. Afterwards, penetrating the intestinal walls, it arrives in the portal vein, where, presumably, it completes its development. After I had despatched these conclusions I continued my researches 
in Gabes and discovered that the larra of an insect (probably a species of ephemerida) was also an efficient intermediary host. With the riew of making control experiments I proceeded to Gafsa, as I had been assured by the principal medical officer of the military hospital of that place that bilharzia disease was endemic there. Here I had access to several cases of indigenous origin. I found that not only is the disease very common in Gafsa in the native population, but that the cases were sometimes of a grave character, such as I had previously frequently met with in Egypt. I may say that my researches in Gafsa confirm the conclusions above detailed in so far as I succeeded in rearing bilharzia embryos in the aquatic larva of an insect; but, strange to say, $I$ did not meet with this insect larva at Gabes; nor did I succeed in finding in Gafsa the larva of the insect nor the crustacean which served me as subjects for my experiments at Gabes. 'Thus I concluded that several kinds of aquatic arthropoda may act as efficient intermediary hosts for bilharzia, whilst molluscs seem to have nothing to do with its life history. I may add that in these intermediary hosts the cysts of bilharzia resemble very closely the asexually generated cysts of young distomes. They are globular, having a double outline and a diameter of from $0.075 \mathrm{~mm}$. to $0.120 \mathrm{~mm}$. If the included parasite be not moving spontaneously, movement may be elicited by adding to the slide a little warm water. The cyst is elastic and compressible, but it is difficult to rupture. $U p$ to the present I have failed to liberate the parasite without completely destroying it. As seen in the cyst it presents no features by which it can be distinguished from other kinds of encysted distomes, with the exception that its integument is firmly and characteristically beaded, just as in the earlier stage of the embryo bilharzia, as described by Cobbold ${ }^{1}$ in 1872, and as I figured it in my first paper ${ }^{2}$ on this parasite in 1874 . I may mention that as regards the anatomy of the embryo bilharzia I can confirm what has quite recently been announced by Railliet, ${ }^{3}$ but which I have long known, that whilst still in ovo it is provided with two pairs of ciliated infundibula, one pair being in the anterior third and one pair in the posterior third of the body. These infundibula are very apparent and are to be best seen by slowly lowering the focus of the microscope whilst examining the embryo. As the larval phase of bilharzia found encapsuled in the intermediate host is, in a biological sense, different from the encysted cercaria phase of the typical distomes - inasmuch as it is a direct encystment of the embryo and not, as in the case of the cercaria of the distomes, the encystment of the asexually generated progeny of a redia developed from an embryo distome, - it is desirable to provide this larval encysted stage of bilharzia with a distinctive name. As the corresponding larval phase of the holostomes-which, as regards this part of their life history, bilharzia closely resembles-is designated "tetracotyle," the term " dicotyle" appears to be appropriate, the bilbarzia larva having only two suckers. The insect aquatic larvæ in which the dicotyle of bilharzia is found encysted have a length of 7-8 mm. and a thickness of about $0.3 \mathrm{~mm}$. The crustacean is about the same size. The magnitude of these intermediary hosts renders it easy by a little attention being paid to the drinking water to preserve a community from infection by bilharzia, as the investigation I have made would tend to show that the drinking water which is obtained from pools, tanks and streams which contain arthropoda is the medium by which this parasite gains access to the human body and it is probably the only medium ; therefore bathing in such water, if the bather should swallow the water, is dangerous. Filtration, even rough filtration through linen, will aftord complete immunity. It will thus be seen that the rules I laid down many years ago for the prevention of bilharzial infection, which were repeated in a paper read by me at the last International Congress of Hygiene (1891), and which appeared in THE LANCET of Aug. 22nd and 29 th, 1891, are still appropriate.

I wish also to note that the discovery of the life history of bilharzia, to wbich I applied myself now more than ten years ago, and which, perhaps, I would have effected much sooner could I have continued my earlier researches in the Khedivial 1 On the Development of bilbarzia bæmatobia, bing a paper read
before the Metropolitan Counties Branch of the British Medical Association on May 17th, 1872. (Brit. Med. Jour.)

2 Ricente intorna alla Bilhas zia Hæmatobia in relazione colla Imaturia Endemica in Egitto. Rendiconto della R. Academia della Scienze di Nannli, 1874.

3 Observations sur l'Embryon du Gynecophorus Hæmatohius Bilhan zia. Par.A. Ralliet, Paris, j892. Bulletin de la Société Zoologique de France, 1892, tome xvii., p. 151
Laboratory at Cairo, gives prophetic significance to the forecast which I made in a paper published in 1884, the concluding words of which I reproduce in their original language: "Non é improbabile che la bilharzia che per tante particolaritá s'allontana dagli altri generi di distomi ofira ancora questa singolarità di avere per ospiti intermediarii animali di classi diverse da quelle che servono allo oviluppo degli altri distomi o quella di compire tutto il suo vilo vitalé in parte libero nell' acqua e in parte parassita in un solo ospite finale e forte senza reproduzione alternante."

Gafsa, Tunis.

\section{ANATOIIY AND PHYSIOLOGY OF THE BILHARZIA OVUM :}

\section{EXTRACT FBON A SCMMARY OF A THESIS ON BILHARZIA} HAMATOBIA.

BY GEORGE SANDISON BROCK, M.D. EDIN.

I wxSH, in the first place, to describe with some minuteness the ovum of the bilharzia. In itself it is both curious and interesting, and it is well worth study; and, as a complete knowledge of it seems to be the only means of putting the question of its identity with that of the Egyptian parasite beyond dispute, I have bestowed a good deal of attention upon the subject. It has been a difficult matter in studying such a delicate object to make out its various parts witb accuracy, and I cannot pretend to have done so; yet I hare been able to find out some important points that, so far as I am aware, have not been noticed by any previous observer. As already mentioned, the ova of the bilharzia have been seen in the ovisac of the mature worm, but I have only been able to study them as they appeared in the urine of patients who were suffering from the disease. In this situation they may, however, be met with in all stages of development, and in enormous numbers. The most immature ova have a simple envelope with a short spine at one end, and they are filled with granules of various sizes. They are (Cobbold) about $\frac{1}{40}$ in. in length, and about To in. in breadth. In older specimens segmentation may be seen in different stages of advancement, and by searching carefully one may trace, step by step, the development from a simple chaotic granular mass through all the stages until the creature finally attains the definite and compara. tively complex structure of the mature embryo. The fully matured ovum (see p. 623) is very similar in shape to a hen's egg and measures from $\frac{1}{150}$ in. to $\frac{1}{6} \sigma$ in. in length and about $\frac{1}{3}$ in. in diameter (all measurements are taken from Cobbold). There is, however, much irregularity to be seen even in mature specimens, both as regards size and shape. Some are a little larger and others are a good deal smaller; whilss some arelong and narrow others have more the shape of a pear or a boy's kite. The outer envelope or shell is so clear and smooth that it scarcely, if at all, interferes with one's view of its contents. By reflected light it appears to be shiny and of a brownish or pinkish neutral tint. It is soft, clelicate, and very thin, but its double contour can be made out under a high power, especially at the narrower end of the egg, at which part a sharp, projecting spine of varying length is usually placed. This spine is often very short and it is sometimes altogether absent. Its average length is about ${ }^{1}$ in. or one-tenth of the total length of the egg. According to Cobbold it is the homologue of a very much larger structure in certain other trematode ova, where it serves as a "holdfast" br which the eggs attach themselves to the body of their host. In the bilharzia its use in this respect, if it has any, must from its straightness and trifling length be very slight. In eggs which have been voided through the rectum it is sais that these spines are often placed laterally. I have never been able to obtain any so passed, but I can confirm Harley's assertion that those from the urinary passages never present this peculiarity. Lining the interior of the shell and intexvening between it and the embryo is a delicate granular membrane. The space between this membrane and the embryo varies much according to the size and state of contraction of the latter, shortly before whose escape it becomes filled with numbers of bright granules which are extruded through certain apertures (which are presently to be described) in the sides of the embryo. These granules exhibit Brownian movements and are often further agitated 